\title{
How Do Spherical Diblock Copolymer Nanoparticles Grow During RAFT Alcoholic Dispersion Polymerization?
}

\author{
E. R. Jones, O. O. Mykhaylyk, M. Semsarilar, M. Boerakker, P. Wyman, S. P. Armes*
}

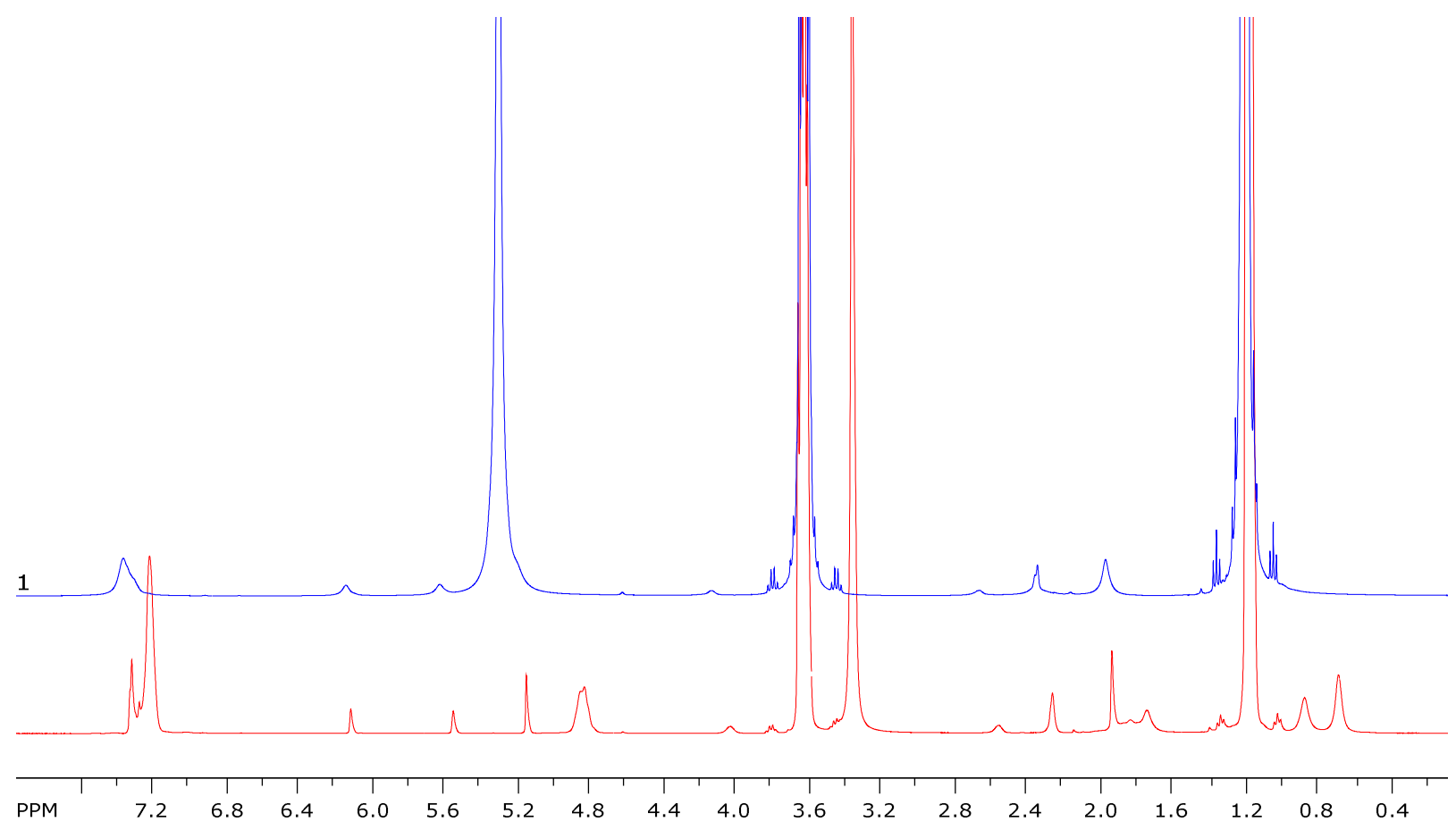

Figure S1. ${ }^{1} \mathrm{H}$ NMR spectra of $\mathrm{PDMA} \mathrm{A}_{94}-\mathrm{PBzMA}_{810}$ recorded in $\mathrm{C}_{2} \mathrm{D}_{5} \mathrm{OD}$ (upper spectrum) or $\mathrm{CD}_{2} \mathrm{Cl}_{2}$ (lower spectrum). The former spectrum gives an apparent composition of $\mathrm{PDMA}_{94}-\mathrm{PBzMA}_{64}$ because the coreforming PBzMA block is only partially solvated in ethanol. In contrast, $\mathrm{CD}_{2} \mathrm{Cl}_{2}$ is a good solvent for both blocks, so the PBzMA peaks are fully solvated in this solvent and the actual block composition can be calculated. In both cases, signals arising from residual BzMA monomer are observed at 5.6 and $6.1 \mathrm{ppm}$, which is expected given the incomplete conversion (81\%) for this synthesis. This unreacted BzMA monomer partially solvates the PBzMA core-forming chains in addition to the $\mathrm{C}_{2} \mathrm{D}_{5} \mathrm{OD}$. However, the apparent block composition calculated above suggests that the degree of solvation is no more than $8 \%$, even in this case. Such ${ }^{1} \mathrm{H}$ NMR analyses were performed for all diblock copolymer nanoparticles described in this paper, with estimated degrees of solvation ranging from 5-13\%.

\section{Spherical micelle model used for SAXS analysis}

In general, the scattering intensity of a system comprising just one type of non-interacting polydisperse objects can be expressed as 
$I(q)=N \int_{0}^{\infty} \ldots \int_{0}^{\infty} F\left(q, r_{1}, \ldots, r_{k}\right)^{2} \Psi\left(r_{1}, \ldots, r_{k}\right) d r_{1} \ldots d r_{k}$

where $F\left(q, r_{1}, \ldots, r_{k}\right)$ is the form factor for the scattering objects, $\Psi\left(r_{1}, \ldots, r_{k}\right)$ is the distribution function, $N$ is the number density per unit volume of the objects and $r_{1}, \ldots, r_{k}$ is a set of $k$ parameters describing their structural morphology. The PDMA ${ }_{94}-\mathrm{PBzMA}_{\mathrm{x}}$ diblock copolymer chains studied in this work self-assemble in ethanol to form spherical nanoparticles (or micelles) (Figure 2). Thus, the form factor in eq S1 can be expressed via an analytical expression previously reported for spherical copolymer micelles. ${ }^{1}$

$F(q)=\left[N \beta_{m c} A_{m c}(q)\right]^{2}+N_{a g g} \beta_{c}^{2} F_{c}\left(q R_{g}\right)+N_{a g g}\left(N_{a g g}-1\right) \beta_{c}^{2} A_{c}^{2}(q)+2 N_{a g g}^{2} \beta_{m c} \beta_{c} A_{m c}(q) A_{c}(q)$

Here the X-ray scattering length contrast for the core block and the corona block is given by $\beta_{m c}=V_{m c}\left(\xi_{m c}-\xi_{s o l}\right)$ and $\beta_{c}=V_{c}\left(\xi_{c}-\xi_{s o l}\right)$, where $\xi_{m c}, \xi_{c}$, and $\xi_{s o l}$ are the X-ray scattering lengths of the core block $\left(\xi_{\text {PBIMA }}=10.67 \times 10^{-10} \mathrm{~cm}^{-2}\right)$, the corona block $\left(\xi_{\text {PDMA }}=10.12 \times 10^{-10} \mathrm{~cm}^{-2}\right)$ and the solvent $\left(\xi_{\text {ethanol }}=7.57 \times 10^{-10} \mathrm{~cm}^{-2}\right)$, respectively. $V_{m c}$ and $V_{c}$ denote the volumes of the core block $\left(V_{P B Z M A x}\right.$, see Table 2$)$ and the corona block $\left(V_{P D M A 94}=22.5 \mathrm{~nm}^{3}\right)$, respectively. These volumes were obtained from $V=\frac{M_{w}}{N_{A} \rho}$ using the molecular weight $\left(M_{w}\right)$ of the corresponding block and the solid-state homopolymer densities determined by helium pycnometry $\left(\rho_{P B Z M A}=1.18 \mathrm{~g} \mathrm{~cm}^{-3}\right.$ and $\left.\rho_{P D M A}=1.09 \mathrm{~g} \mathrm{~cm}^{-3}\right)$. The spherical form factor amplitude is used for the amplitude of the micelle core self-term

$$
A_{m c}(q)=\frac{3\left[\sin \left(q R_{s}\right)-q R_{s} \cos \left(q R_{s}\right)\right]}{\left(q R_{s}\right)^{3}}
$$

where $R_{s}$ is the radius of the spherical micelle core. The mean aggregation number for the spherical micelles is given by $N_{\text {agg }}=\left(1-x_{s o l}\right) \frac{4 \pi R_{s}^{3}}{3 V_{m c}}$, where $x_{s o l}$ is the solvent fraction in the micelle core. The selfcorrelation term of the corona block is described by the Debye function:

$$
F_{c}\left(q R_{g}\right)=\frac{2\left[\exp \left(-q^{2} R_{g}^{2}\right)-1+q^{2} R_{g}^{2}\right]}{q^{4} R_{g}^{4}}
$$

where $R_{g}$ is the radius of gyration of the corona block. For diblock copolymers with a relatively short PBzMA block DP, the corona contribution to the scattering is comparable to the scattering from the micelle core [i.e. $\mathrm{PDMA}_{94}-\mathrm{PBzMA}_{100}$ with $\left(\beta_{c} / \beta_{m c}\right)^{2} \approx 0.26$ ]. Thus, in accordance with previous work, ${ }^{2}$ the amplitude of the corona self-term was obtained from a normalized Fourier transform of the radial density distribution function of the $\mathrm{PDMA}_{94}$ coronal chains in the micelle. 
$A_{c}(q)=\frac{\int_{R_{s}}^{R_{s}+2 s} \mu_{c}(r) \frac{\sin (q r)}{q r} r^{2} d r}{\int_{R_{s}}^{R_{s}+2 s} \mu_{c}(r) r^{2} d r}$

The radial profile, $\mu_{c}(r)$, is expressed by a linear combination of two cubic $b$ splines with two fitting parameters $s$ and $a$ corresponding to the width of the profile and the weight coefficient, respectively. ${ }^{3}$ The precise analytical expression of the integration applied in the SAXS analysis is not given in the reference but it can be obtained by using a mathematical software package such as Maple or MatLab. An approximate integration can also be found elsewhere. ${ }^{4}$ In accordance with previous studies ${ }^{2-4}$ a confinement $s=2 R_{g}$ was introduced into the model. It is known that $a$ tends to zero for this condition, so it was assumed that $a=0$. For the form factor given by eq $\mathrm{S} 2$, no penetration of the corona blocks into the micelle cores and a sharp, non-sigmoidal interface between the blocks was assumed. A polydispersity for the spherical micelle core radius $\left(R_{s}\right)$, expressed as a Gaussian distribution, is considered for the spherical micelles in eq S1:

$\Psi\left(r_{1}\right)=\frac{1}{\sqrt{2 \pi \sigma_{R s}^{2}}} e^{-\frac{\left(r_{1}-R_{s}\right)^{2}}{2 \sigma_{R S}^{2}}}$

where $\sigma_{R s}$ is the standard deviation for $R_{s}$. The number density per unit volume in eq $\mathrm{S} 1$ is expressed as:

$$
N=\frac{c}{\int_{0}^{\infty} V\left(r_{1}\right) \Psi\left(r_{1}\right) d r_{1}}
$$

where $c$ is the total copolymer volume fraction in the spherical micelles and $V\left(r_{1}\right)$ is the total copolymer volume in a spherical micelle $\left[V\left(r_{1}\right)=\left(V_{m c}+V_{c}\right) N_{a g g}\left(r_{1}\right)\right]$. Thus, the overall number of structural parameters for the spherical micelle model described by eq S1 and eq S2 is seven $\left(R_{s}, \sigma_{R s}, x_{s o l}, R_{g}, s, a\right.$ and $c$ ). Four of these seven parameters are determined independently, leaving just three variable parameters available for modelling.

\section{Differential refractive index measurements}

Differential refractive index $\left(\mathrm{dn} / \mathrm{dc}\right.$ ) values were obtained for each of the $\mathrm{PDMA}_{94}-\mathrm{PBzMA}_{\mathrm{x}}$ diblock copolymer spherical nanoparticles using a Wyatt Optilab T-rEX differential refractometer. Nanoparticle dispersions were prepared in ethanol at copolymer concentrations of $0.1,0.2,0.3,0.4$ and $0.5 \mathrm{mg} \mathrm{mL}^{-1}$ and then injected consecutively into the instrument (lowest concentration first). Figure S1 illustrates a typical data set obtained for $\mathrm{PDMA}_{94}-\mathrm{PBzMA}_{392}$ nanoparticles. Plotting a calibration graph of $\mathrm{dRI}$ against copolymer concentration allows the $\mathrm{dn} / \mathrm{dc}$ to be calculated directly from the linear gradient (see inset in Figure S1). 


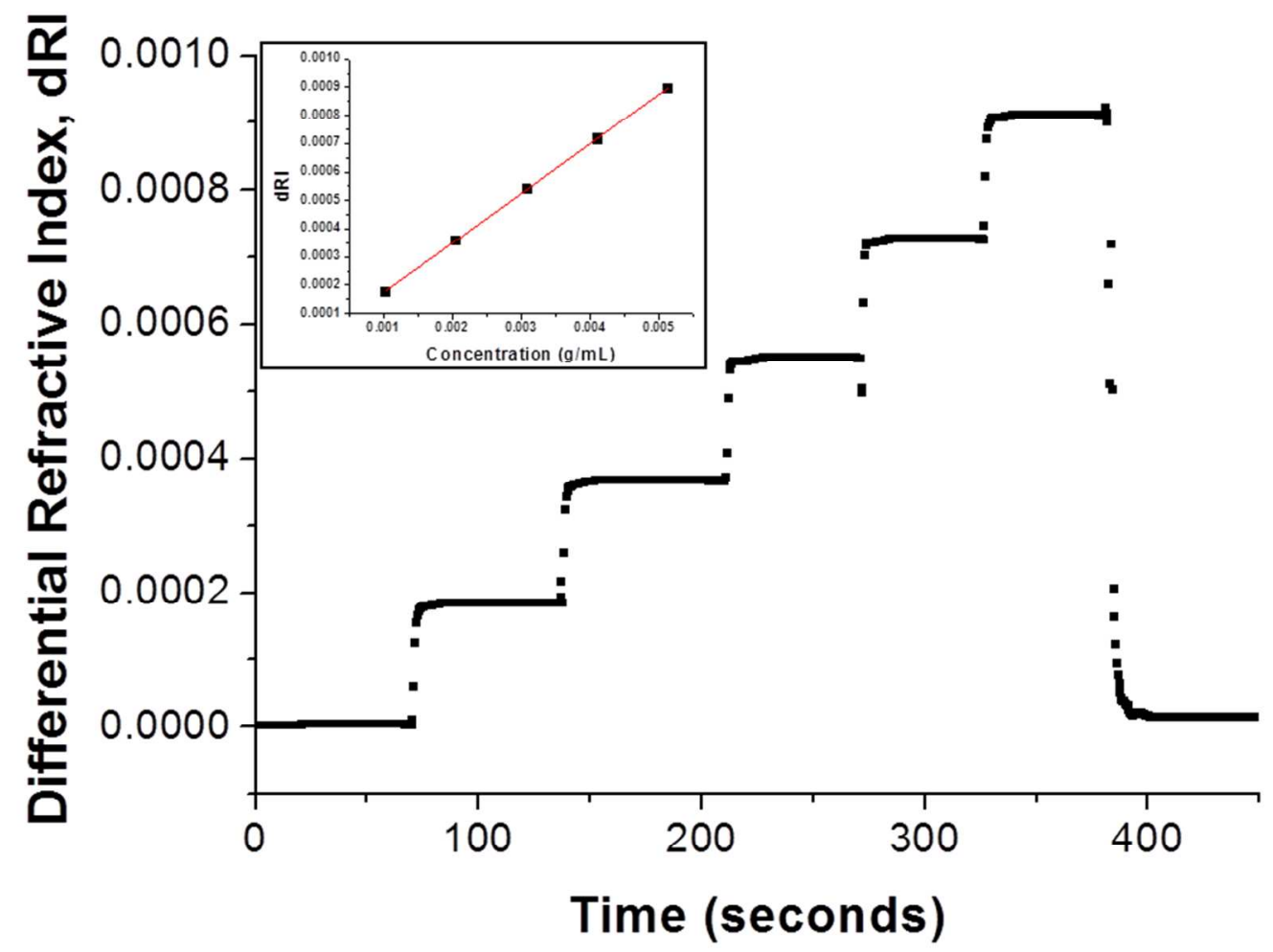

Figure S2. Change in refractive index, dRI, for a series of five PDMA $94-\mathrm{PBzMA}_{392}$ nanoparticle concentrations determined using a Wyatt Optilab T-rEX instrument equipped with Astra software. Inset is the plot of dRI against copolymer concentration, which was subsequently used to calculate the differential refractive index $(\mathrm{dn} / \mathrm{dc})$ for these nanoparticles.

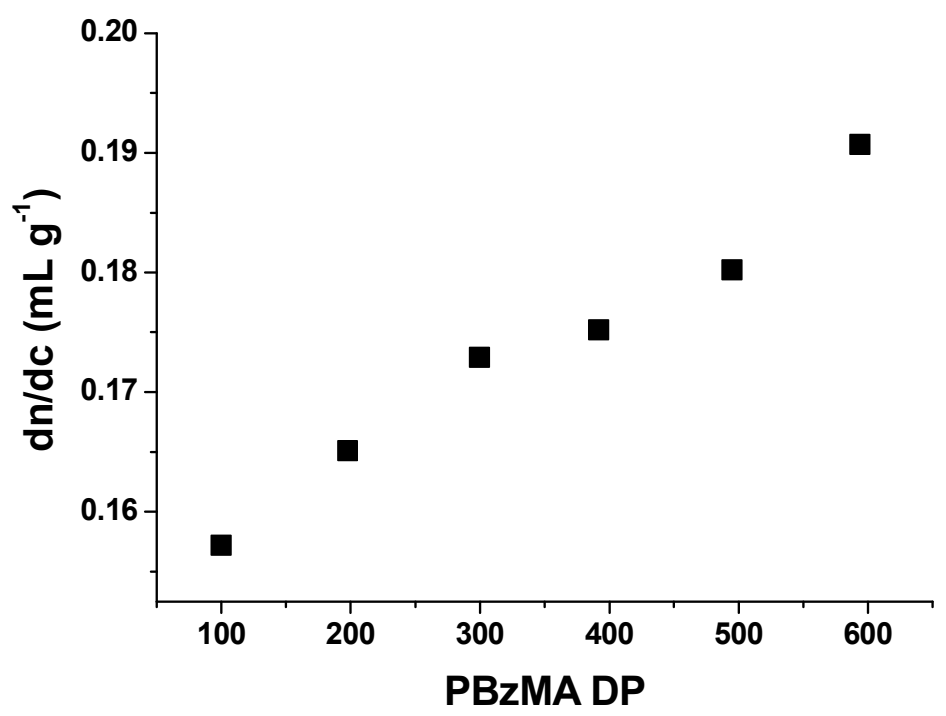

Figure S3. Summary of $d n / d c$ values calculated for a series of $P D M A_{94}-P B z M A_{x}$ diblock copolymer nanoparticles using a Wyatt Optilab T-rEX differential refractometer and Astra software (provided by the manufacturer). 


\section{Molecular weight determination from MALLS data}

Debye formalism: $\frac{R_{\theta}}{K c}=M P(\theta)-2 A_{2} c M^{2} P^{2}(\theta)$

Zimm formalism (reciprocal method): $\frac{K c}{R_{\theta}}=\frac{1}{M P(\theta)}+2 A_{2} C$

Berry formalism (square root method): $\sqrt{\frac{K c}{R_{\theta}}}=\frac{1}{\sqrt{M P(\theta)}}+A_{2} c \sqrt{M P(\theta)}$

For spheres: $\quad P(\theta)=\left(\left(\frac{3}{x^{3}}\right)(\sin x-x \cos x)\right)^{2}$

Where: $\quad x=2 \pi\left(\frac{D}{\lambda}\right) \sin \left(\frac{\theta}{2}\right) \quad(\mathrm{D}=$ sphere diameter $)$

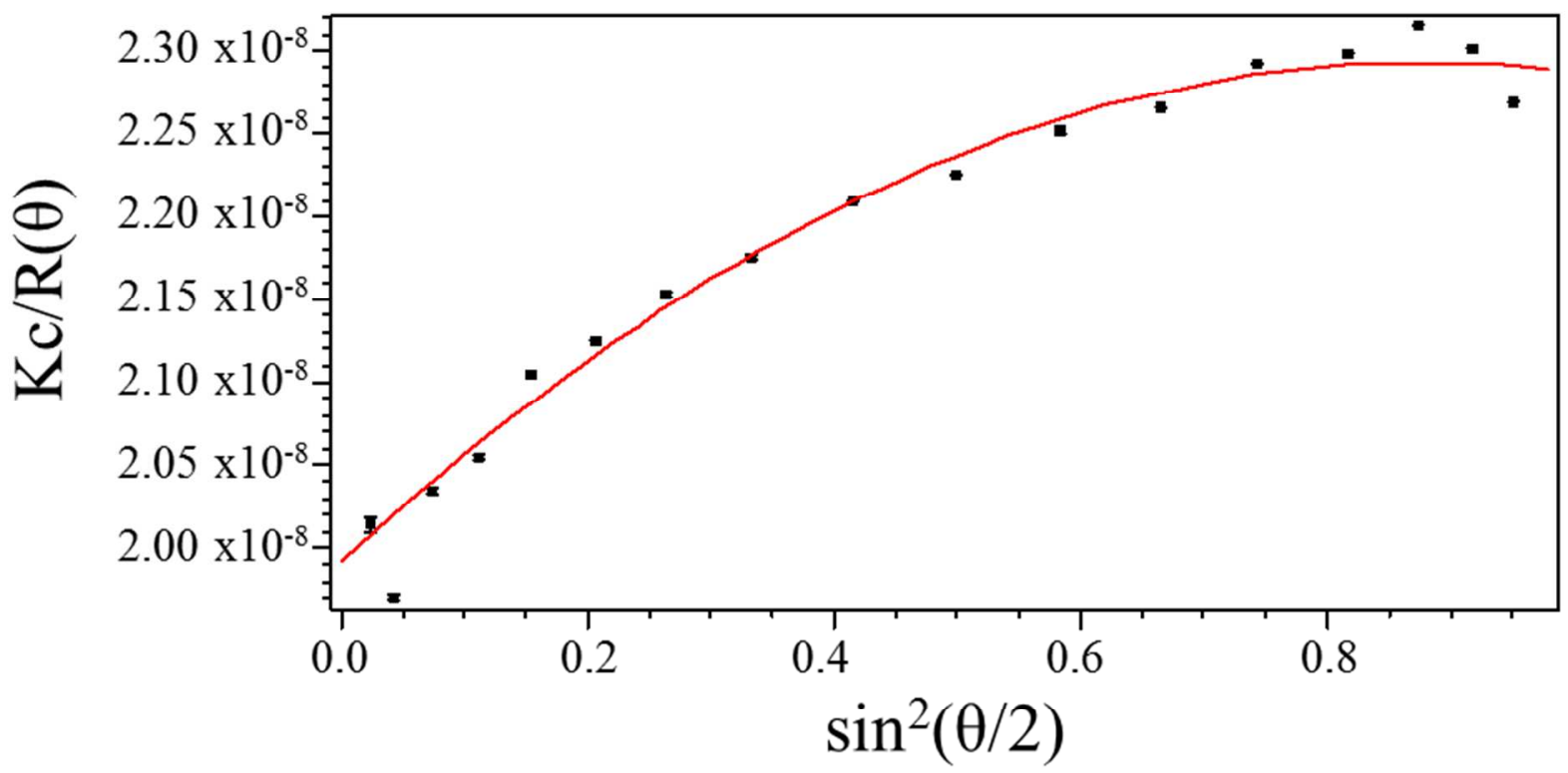

Figure S4. Representative MALLS plot using the Zimm formalism for light scattering data obtained for $\mathrm{PDMA}_{94}-\mathrm{PBzMA}_{392}$ diblock copolymer micelles dispersed in ethanol. The weight-average molecular weight $\left(M_{\mathrm{w}}\right)$ of these micelles was determined to be $5.001 \times 10^{7} \mathrm{~g} \mathrm{~mol}^{-1}$, which indicates a micelle aggregation number, $N_{\text {agg, }}$ of 443 . 


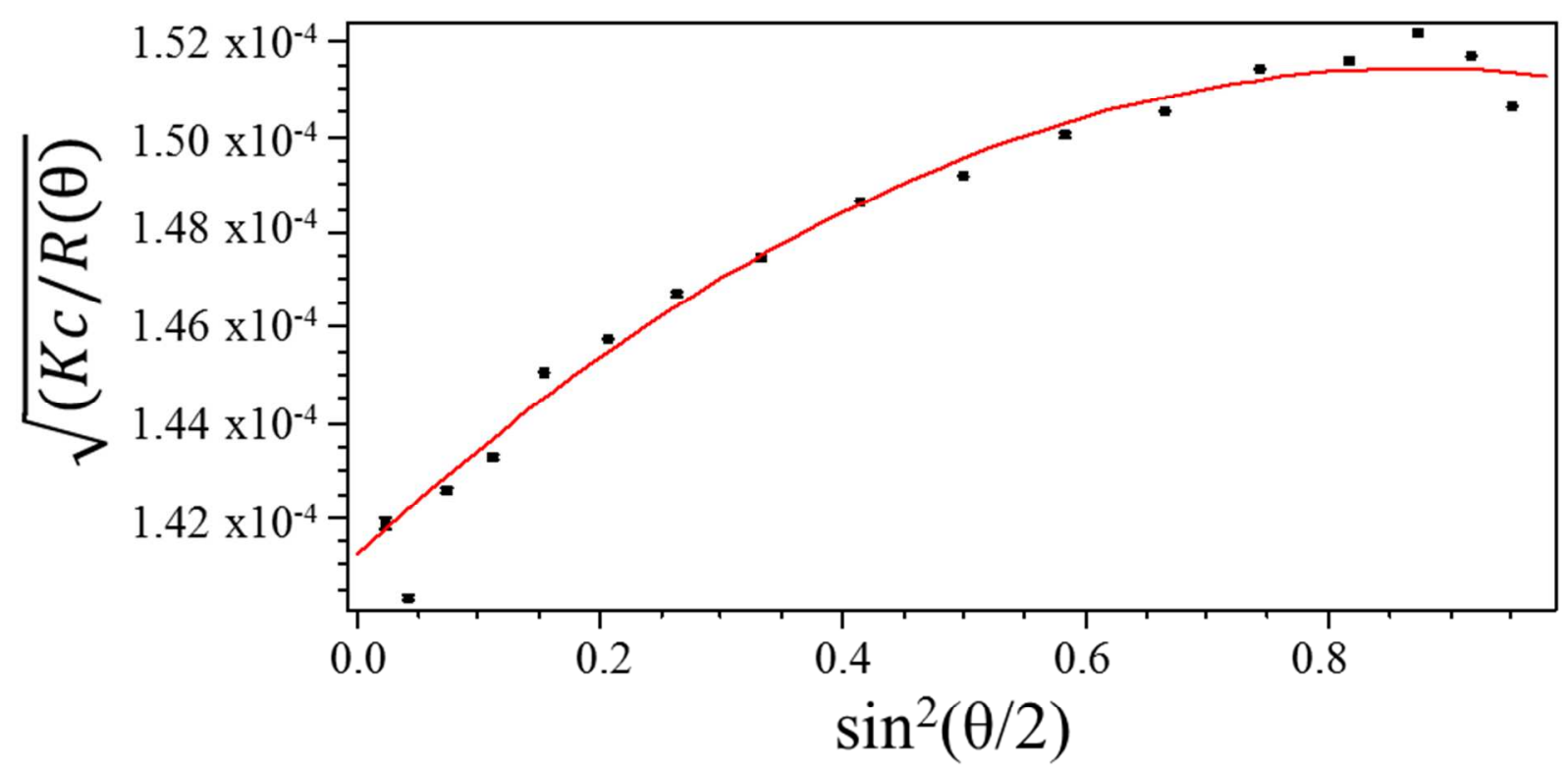

Figure S5. Representative MALLS plot obtained using the Berry formalism to analyse light scattering data obtained for $\mathrm{PDMA}_{94}-\mathrm{PBzMA}_{392}$ diblock copolymer nanoparticles dispersed in ethanol. The weightaverage molecular weight $\left(M_{\mathrm{w}}\right)$ for these micelles was determined to be $5.01 \times 10^{7} \mathrm{~g} \mathrm{~mol}^{-1}$, which indicates a mean micelle aggregation number, $N_{\mathrm{agg}}$ of 444 .

Table S1. Weight-average molecular weights determined by MALLS for PDMA P4 $^{-} \mathrm{PBzMA}_{\mathrm{x}}$ diblock copolymer nanoparticles using the three most commonly used analytical methods, and the corresponding mean micelle aggregation numbers.

\begin{tabular}{|c|c|c|c|}
\hline Composition & Analysis Method & $\mathbf{M}_{\mathbf{w}}$ & $\mathbf{N}_{\text {agg }}$ \\
\hline \multirow{3}{*}{$\mathrm{PDMA}_{94}-\mathrm{PBz} \mathrm{MA}_{100}$} & Zimm & $5.83 \times 10^{6}$ & 133 \\
\hline & Debye & $5.81 \times 10^{6}$ & 132 \\
\hline & Berry & $5.82 \times 10^{6}$ & 133 \\
\hline \multirow{3}{*}{$\mathrm{PDMA}_{94}-\mathrm{PBz} \mathrm{MA}_{198}$} & Zimm & $1.77 \times 10^{7}$ & 268 \\
\hline & Debye & $1.76 \times 10^{7}$ & 266 \\
\hline & Berry & $1.77 \times 10^{7}$ & 268 \\
\hline \multirow{3}{*}{$\mathrm{PDMA}_{94}-\mathrm{PBz} \mathrm{MA}_{392}$} & Zimm & $5.01 \times 10^{7}$ & 444 \\
\hline & Debye & $4.98 \times 10^{7}$ & 441 \\
\hline & Berry & $5.00 \times 10^{7}$ & 443 \\
\hline \multirow{3}{*}{$\mathrm{PDMA}_{94}-\mathrm{PBz} \mathrm{MA}_{495}$} & Zimm & $7.35 \times 10^{7}$ & 532 \\
\hline & Debye & $7.30 \times 10^{7}$ & 528 \\
\hline & Berry & $7.37 \times 10^{7}$ & 533 \\
\hline \multirow{3}{*}{$\mathrm{PDMA}_{94}-\mathrm{PBz} \mathrm{MA}_{594}$} & Zimm & $1.01 \times 10^{8}$ & 685 \\
\hline & Debye & $9.90 \times 10^{7}$ & 672 \\
\hline & Berry & $9.96 \times 10^{7}$ & 676 \\
\hline
\end{tabular}


Figure S6. MALLS plots obtained using the Debye formalism for each of the PDMA ${ }_{94}-\mathrm{PBz}_{\mathrm{MA}} \mathrm{d}_{\mathrm{x}}$ diblock copolymer nanoparticles dispersed in ethanol. The associated weight-average molecular weights $\left(M_{\mathrm{w}}\right)$ and calculated mean aggregation numbers, $N_{\text {agg, }}$ are listed in Table S1.
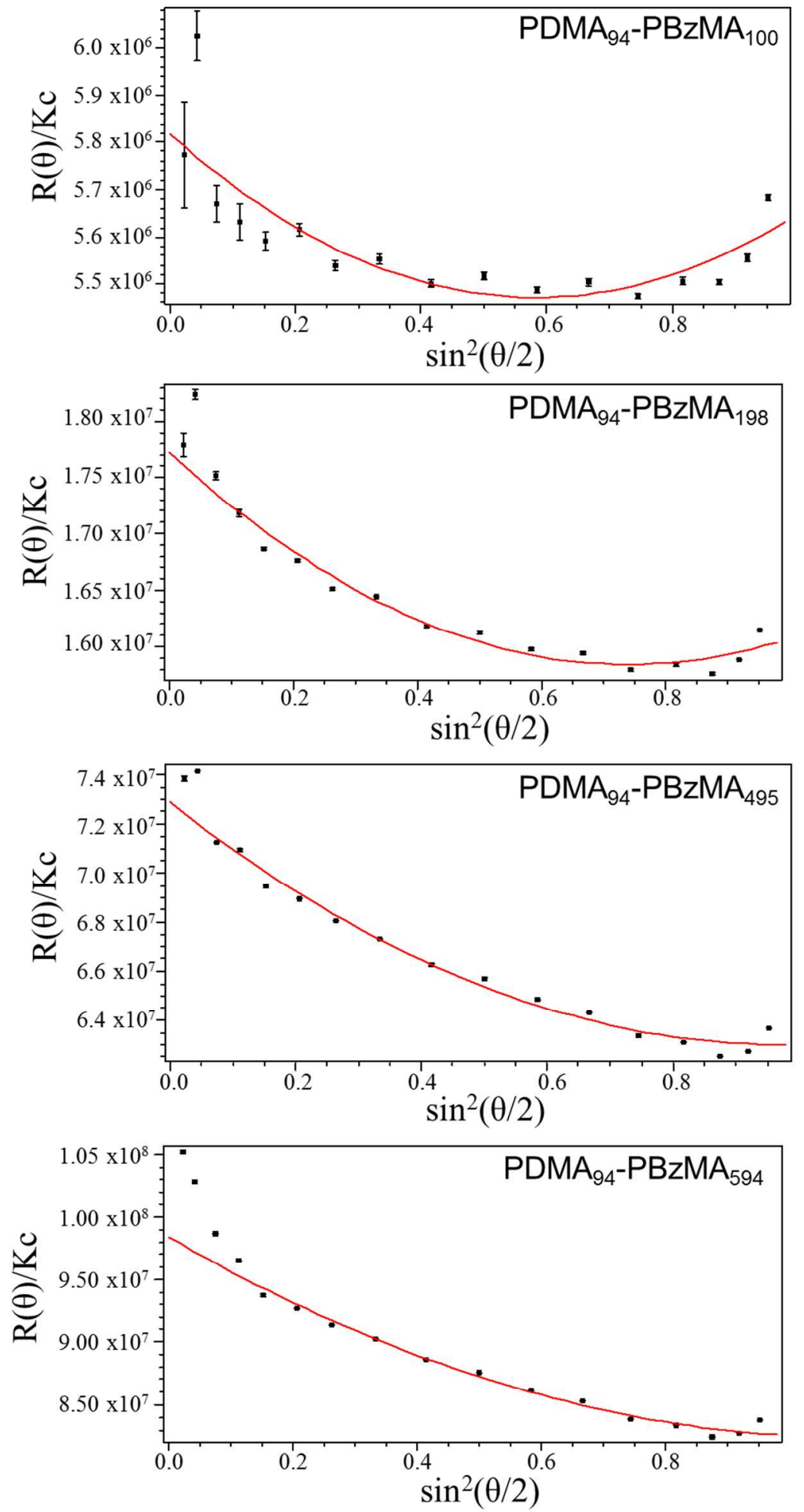


\section{References}

(1) Pedersen, J. S. J. Appl. Crystallogr. 2000, 33, 637.

(2) Warren, N. J.; Mykhaylyk, O. O.; Ryan, A. J.; Williams, M.; Doussineau, T.; Dugourd, P.; Antoine, R.; Portale, G.; Armes, S. P. J. Am. Chem. Soc. 2015, 137, 1929.

(3) Pedersen, J. S.; Gerstenberg, M. C. Colloid Surf. A-Physicochem. Eng. Asp. 2003, 213, 175.

(4) Pedersen, J. S.; Svaneborg, C.; Almdal, K.; Hamley, I. W.; Young, R. N. Macromolecules 2003, 36, 416. 